\title{
Generalized Trust and Social Selection Process
}

\author{
Tasuku Igarashi ${ }^{1 *}$ and Taro Hirashima ${ }^{2}$ \\ ${ }^{1}$ Graduate School of Education and Human Development, Nagoya University, Nagoya, Japan, ${ }^{2}$ Department of Psychology, Aichi \\ Shukutoku University, Nagakute, Japan
}

\section{OPEN ACCESS}

Edited by:

Monica Thiel,

University of International Business and Economics, China

Reviewed by:

Sebastian Pink,

Universität Mannheim, Germany

João R. Daniel,

William James Center for Research,

Portugal

*Correspondence:

Tasuku Igarash

igarashi.tasuku@d.mbox.nagoya-

u.ac.jp

Specialty section: This article was submitted to

Organizational Psychology,

a section of the journal

Frontiers in Communication

Received: 12 February 2021 Accepted: 26 April 2021

Published: 10 May 2021

Citation:

Igarashi T and Hirashima T (2021)

Generalized Trust and Social

Selection Process.

Front. Commun. 6:667082.

do: $10.3389 /$ fcomm.2021.667082
Generalized trust relieves individuals in a socially uncertain situation from dyadic constraints of existing ties and helps them change ties with other individuals to acquire better resources. However, much evidence in the emancipation role of generalized trust as a booster of new relationship formation has been limited to laboratory experiments or cross-sectional surveys. We conducted a four-wave longitudinal survey to test whether individuals high in generalized trust actively switch ties and form open triads in dynamic social networks. Stochastic ActorOriented Models were employed to analyze structural changes in advice and personal discussion networks among first-year undergraduates. Results showed the predicted patterns of social selection processes based on generalized trust when the dynamics of the two networks were analyzed simultaneously: only in the advice network, individuals high in generalized trust tended to terminate existing ties, create new ties, and show a decreasing trend toward forming close triads when the degree of local clustering was large. Effective tie-formation strategies of individuals high in generalized trust in a multiplex network structure are discussed.

Keywords: social networks, generalized trust, social selection process, multiplex networks, stochastic actororiented model

\section{INTRODUCTION}

\section{Emancipation Theory of Trust}

Trust in others is fundamental to sustaining social life. Individuals in social networks, often called actors, can overcome social difficulties by trusting other individuals to share and exchange diverse resources. Trust is an important basis for increasing social capital (Coleman, 1988; Sampson et al., 1997; Putnam, 2000) and results in new encounters in a situation where a social network is changeable and expandable (Burt, 1993; Igarashi et al., 2008). Previous research has revealed that actors who trust other actors can cooperate with strangers in a social network to coordinate mutual benefits across diverse social settings, such as in laboratory experiments (Yamagishi, 2011), work organizations (Lusher et al., 2012), and online social networking sites (Valenzuela et al., 2009).

The primary goal of this study is to discover the way in which a social network is constructed and renewed based on the mechanism of trust in social relationships. In particular, this study focuses on the concept of generalized trust, or an unconditional expectation that people will be benevolent. According to Yamagishi and Yamagishi (1994), generalized trust refers to an expectation of the benign intentions of other people in general. In other words, no specific and existing interpersonal relationship is construed in the framework. The psychological concept indicates the "default" degree to which people trust others in general when no information about their cooperativeness is provided. Generalized trust at the attitude level is regarded as stable (Yamagishi et al., 1998) and has sufficient validity in the prediction of trustful behavior toward strangers (Yamagishi et al., 2015). 
Trustful actors, or those high in generalized trust tend to judge strangers to be trustworthy (Yamagishi, 2011). However, this does not mean that individuals high in generalized trust are more gullible than those low in generalized trust. Trust is associated with a sort of social intelligence, or the ability to avoid risks of interacting with non-cooperative others. Social intelligence leads people to success in interpersonal communication under social uncertainty, defined as a situation in which little information is available for the assessment of others' intention to cooperate. In fact, generalized trust increases the possibility to detect others' trustworthiness from nonverbal cues (Bonnefon et al., 2013). This detection ability has its roots in the brain function related to mindreading (Watabe et al., 2011) and is also effective in facilitating cooperation in everyday social situations (Hashimoto et al., 2020). In a social network, generalized trust works as a significant psychological characteristic enabling one to actively create new social relationships with strangers beyond existing relationships.

Using terminology borrowed from economics, the emancipation theory of trust (Yamagishi and Yamagishi, 1994; Yamagishi, 2011) assumes that individuals high in generalized trust employ a tie-selection strategy based on the careful consideration of opportunity costs provided by the existing social ties that surround them. Under a condition where social networks are changeable, individuals high in generalized trust are motivated to search for the social tie that is the most valuable one in which to "invest" their own resources to achieve mutual cooperation through that tie. The resources can be diverse from economic to psychological (Foa and Foa, 1976), and the investment includes looking for the best possible partners to support them and finding collaborators with joint interests. To maximize their resources, individuals high in generalized trust always compare the rewards received from an existing tie to potential rewards from an available alternative tie. If the latter exceeds the former, then individuals high in generalized trust may disengage from the existing tie and develop a new connection to the alternative tie due to the limited time available to spend on communication (Dunbar et al., 2009).

According to Yamagishi et al. (1999), the greater the cognitive cost individuals high in generalized trust pay for detecting others' trustworthiness, the greater the returns they can expect from a more cooperative partner they can find in their social network. They are sensitive to the possibility of being exploited by spiteful strangers and exercise the detection ability to avoid harmful scenarios. Therefore, individuals high in generalized trust are emancipated from commitment relationships in the sense that they can disengage from existing social ties when they find alternative ties that may provide better outcomes in exchange for their cognitive investment to detect others' trustworthiness.

In contrast, individuals low in generalized trust are vigilant and avoid the risk of being left high and dry by others. Even so, external factors such as attendance at social events would help them form new relationships. Once they are connected with someone with whom they can build a "comfort zone," they are not strongly motivated to expand their social circle outside it and are less sensitive to the trustworthiness of strangers. Consequently, they are likely to insist on remaining in commitment relationships, even if they will miss an opportunity to get better rewards outside their existing social ties.

The emancipation theory of trust has been validated by 1) game-theoretical approaches, including repeated interactions among a relatively small number of game players (Yamagishi et al., 1998) and selective tie-formation and termination processes in an uncertain situation (Hayashi and Yamagishi, 1998), 2) a correlational study of the relationships between a generalized trust score and psychological scales measuring well-being and interpersonal orientations among undergraduates (Yoshimoto and Hasegawa, 2017), and 3) nation-level large cross-sectional research on social support exchange (Ishiguro and Okamoto, 2013). Nevertheless, no empirical support for the theory has been obtained in a framework of longitudinal social network dynamics. There is a consistent tendency for individuals high in generalized trust to be free from the burden of existing social relationships and motivated to maximize their resources by forming new connections. Thus, this study predicts that generalized trust promotes the termination of existing ties and the creation of new ties in social networks over time.

\section{Social Selection Process}

To examine the patterns of tie formation in social networks, it is helpful to introduce the concept of social selection processes. Social selection processes refer to the ways in which actors' characteristics (i.e., attributes and attitudes) affect network formation and changes in a given social structure (Erickson, 1988).

The current study predicts that individuals high in generalized trust pursue the formation of open triads in social networks. Open triads are intransitive triangles where actor $\mathrm{A}$ has connections to actors $\mathrm{B}$ and $\mathrm{C}$, but actors $\mathrm{B}$ and $\mathrm{C}$ have no direct connection. Empirically, open triads are not frequently observed because triads tend to converge on closure, in which actors connect with each other in small steps as a reflection of commitment and order. Although the closure principle in triads is grounded in their social nature (Davis, 1970), open triads are still observed in networks where actors are tied through weak emotional bonds (Granovetter, 1973), which enable them to access diverse information or advice in a more efficient way (Burt, 1993; McEvily and Zaheer, 1999). The selective tie formation strategy would be observed only in those who have strong motivation to be autonomous in the network, like individuals high in generalized trust. If individuals high in generalized trust try to maximize their efficiency in accessing resources, they will be selective in tie formation, while avoiding redundant tie formation with those who are located at a two-step distance from them. Being embedded in open triads provides not only personal benefits to actors who are able to gain access to a variety of knowledge but also collective benefits to the network as a whole in terms of efficiency in resource flow. These benefits would work as strong incentives for individuals high in generalized trust to retain the local open structure.

A dyadic selection process also involves the mechanism of homophily, a tendency for similar actors to have ties to each other. Homophily is a tie-formation process among those who have similar status (status homophily: the same role in a group) or 
attitudes (value homophily: similar interests in a topic) (McPherson et al., 2001). Previous findings of the homophily of generalized trust in the field of economic game experiments are controversial. Yamagishi (2011) argues that trustfulness, or generalized trust, is a trait for "selecting" other actors to acquire better resources, whereas trustworthiness is a trait for being "selected" by other actors for the sake of the goodness of the actor (p. 70). On the one hand, trustfulness and trustworthiness do not perfectly overlap. Actors regarded as being trustworthy by other actors do not always behave in a trustful manner (Snijders and Keren, 2001). We could therefore draw a prediction that no tendency of homophily is found for generalized trust. On the other hand, individuals high in generalized trust are selective in communicating with other individuals high in generalized trust. Several studies have shown that trustful behavior is associated with trustworthiness (also Yamagishi and Yamagishi, 1994; Bravo et al., 2012) and that cooperators tend to form groups based on reciprocal nominations in dynamic social networks in which actors are allowed to change ties (Rand et al., 2011). These findings could verify another prediction about homophily in generalized trust: the selection process of individuals high in generalized trust fosters the creation of circles among individuals high in generalized trust, and as a sequel to this, individuals low in generalized trust are constrained to forming ties with other individuals who are also low in trust. Considering the incompatible evidence, the current study explores whether the tie-formation process based on generalized trust follows the principle of homophily.

\section{Types of Sample and Networks}

The current research measured advice and personal discussion networks in a first-year undergraduate sample to test the theory. Adopting a first-year undergraduate sample is a common practice to find significant psychological and interpersonal factors that affect tie-formation processes in the field of social network research (van de Bunt et al., 1999). At the beginning of the first semester, first-year undergraduates are faced with separation from intimate friends and need to develop new social networks with strangers at the university in order to adjust to their surroundings. There is no doubt that university enrollment is one of the most significant opportunities for individuals to reconstruct social networks in accordance with their personal attributes and attitudes.

The application of advice and personal discussion networks in the current research directly follows Yamagishi's (2011) notion about Granovetter's (1973) classification of weak ties (casual relationships with less frequent contacts) and strong ties (stable and close relationships) and their proximity to lowand high-commitment relationships, respectively (p. 70). A group of individuals gathered to seek a wide range of advice/ information would regard their relationships not as mutually controlling but as open and autonomous, whereas those close to each other would regard their relationships as loyal and conscientious. Based on the emancipation theory of trust, it is reasonable to assume that the role of generalized trust in tie formation is more likely to be prevalent in advice/information networks than in personal discussion networks, the former of which is supposed to be changeable and contain common valuable resources in their structure, while the latter is supposed to be stable over time and give access to resources unique to specific exchange partners.

At the time of school transition, first-year undergraduates are faced with a matter of socialization, or an individual-level process of internalizing attitudes, customs, and knowledge that are commonly shared in a given group or society (Weidman, 1989). To access information in an efficient way, it could be beneficial for them to form a greater number of advice-seeking ties and avoid redundancy in the network in order to maintain a diversity of information. It is therefore predicted that generalized trust is useful to create ties to acquire better advice/information for academic success.

Meanwhile, people deemed close friends are well known to each other, linked through frequent contact and strong emotional bonds. One of the basic communication behaviors widely found in intimate relationships is self-disclosure. Self-disclosure is generally reciprocal in close ties and functions not only to increase closeness with a friend but also to decrease the risk of betrayal by the friend after exchanging and sharing secrets. In this context, commitment to particular others (i.e., loyalty and devotion with feelings of safety and assurance; Yamagishi and Yamagishi, 1994) works as a glue in social ties with strong emotional bonds in lieu of generalized trust. For the fulfillment of the need to belong (Baumeister and Leary, 1995), it is wiser for people to stabilize close ties through selfdisclosure based on commitment than to frequently seek opportunities to meet better friends based on generalized trust. Hence, we can draw a prediction that generalized trust does not contribute to the formation of personal discussion ties.

Taking into account the multiplexity of advice and personal discussion networks, it is often the case that people seek advice from those with whom they have already become intimate, and vice versa. However, the supposition would be less important for individuals high in generalized trust because they can be free from being bound to existing relationships and can seek better opportunities for advice outside a given social circle. A decreased impact of personal discussion ties on the formation of advice-seeking ties in the same dyads is thus expected to be found among individuals high in generalized trust.

\section{Hypotheses}

To summarize, the current research examines the following hypotheses to elaborate the dynamics of tie formation based on generalized trust:

Hypothesis 1: Generalized trust is positively related to the tendency to terminate existing ties for advice seeking.

Hypothesis 2: Generalized trust is positively related to the tendency to create new ties for advice seeking.

Hypothesis 3: Generalized trust is positively related to the tendency to form open triads for advice seeking.

Hypothesis 4: Generalized trust is positively related to the tendency to diminish the importance of personal discussion ties when forming advice-seeking ties in the same dyads.

The first three hypotheses should be confirmed not in the personal discussion network but in the advice network. 
Hypotheses 1 and 2 are directly drawn from Yamagishi's original theory (Yamagishi, 2011). Hypothesis 3 includes the interaction between actor disposition and network position. Hypothesis 4 is drawn from the multiplexity of advice and personal discussion networks, each of which represents a low- and high-commitment relationship. Led by the homophily principle, the current study also explores the possibility that individuals high in generalized trust show a preference for other individuals high in generalized trust.

\section{MATERIALS AND METHODS}

\section{Data}

The research was approved by the Ethical Review Board of the Graduate School of Education and Human Development, Nagoya University (\#15-735). The data was collected in Japan as part of a longitudinal research project. This year-long survey was conducted in 2016 to measure psychological and social network characteristics of first-year undergraduates in an education department of a university located in Aichi prefecture (central Japan). All 73 first-year undergraduates in the department agreed to participate in the study $(23$ males and 50 females; $M_{\text {age }}=18.2$ years old). The survey was conducted at four points in time: the 3rd (the beginning of the spring [first] semester: Wave 1), the 12th (the end of the spring semester: Wave 2 ), the 28th (the beginning of the fall [second] semester: Wave 3) and the 41st (the end of the fall semester: Wave 4) weeks of the participants' first academic year after matriculation.

\section{Procedure}

A research assistant attended a new student orientation at the beginning of the spring semester and asked attendees to take part in the survey via LINE (a popular social networking service in Japan) in exchange for a meal voucher (JPY 300) as remuneration at each wave. The electronic consent form was obtained from all participants at that time. Participants were informed that their responses were treated confidentially and that they could discontinue participation in the survey at any time. Participants used their smartphones to answer questions set up on an online platform (Qualtrics) when they had time. The research assistant sent a regular reminder to participants at each wave via LINE to increase response rates. In the survey, demographic and individual measures (generalized trust and personalities) were presented first, followed by network measures.

\section{Measures}

\section{Advice and Personal Discussion Networks}

Advice and personal discussion social networks were collected through survey questions to differentiate between the two types of ties in the same dyad. The advice-seeking network was measured by the question, "Of persons in the department, from whom do you receive information about the department's course subjects, such as teachers' personalities, the content of subjects, details for coursework, and how to complete homework assignments?" The personal discussion network was measured by the question, "Of persons in the department, with whom can you discuss personal matters?" At each of the four waves, participants listed as many names of other students in the same department as possible (i.e., open-ended nominations). The order of presenting the questions was randomized across participants.

\section{Generalized Trust}

Generalized trust was measured at Wave 1 as a continuous variable by the General Trust Scale (Yamagishi and Yamagishi, 1994). The scale consists of six items ("Most people are basically honest," "I am trustful," "Most people are basically good and kind," "Most people are trustful of others," "Most people are trustworthy," and "Most people will respond in kind when they are trusted by others") to assess the degree of expectation of others' trustworthiness in general. Participants answered each item on a 5-point Likert scale (1: strongly disagree to 5: strongly agree). The score was summed over the six items, ranging from 6 to $30(\alpha=0.88 ; M=18.91, S D=4.74)$. A higher score indicates a stronger expectation of others' trustworthiness. Previous literature has revealed that connections through social networks are not associated with the change in generalized trust over time (Sturgis et al., 2015). We followed the empirical finding and regarded generalized trust as stable in a year-long period.

\section{Control Variables}

In order to purify the effect of generalized trust on social selection processes, it is necessary to control the actor- (individual-) and dyad-level effects as extraneous variables on network formation (Snijders, 2017). This study includes sex and personalities at the actor-level control variables and acquaintanceship and renown networks at the dyad-level control variables. The measures of all control variables are reported in Supplementary Material.

\section{Analytic Plan}

This study uses the stochastic actor-oriented model (SAOM) for network dynamics (Snijders, 1996) to capture these tendencies of social network formation among individuals high in generalized trust. SAOM is a powerful statistical tool for analyzing longitudinal data to understand the dynamic processes of network formation. In SAOM, actors are assumed to make changes to their outgoing ties sequentially, resulting in the network changing incrementally. The model is defined by the rate function (modeling the frequency of an actor's opportunity to make a change to the network) and the objective function (modeling the types of changes). The times at which actors are given opportunities to change their ties are modeled using independent exponential distributions with rates that may depend on network properties and actor attributes (e.g., generalized trust). Conditional on an actor being given the opportunity to change one outgoing tie, the preferences for different options are modeled using a conditional logistic model with probabilities proportional to (the exponentiated) objective functions evaluated for each alternative change. The objective function incorporates the current state of ties based on actor attributes and network structures included in the model. In other words, the dependent variable of SAOM indicates the change of ties in a whole social network, such as 0 (tie 
TABLE 1 | Descriptive statistics of advice and personal discussion networks.

\begin{tabular}{|c|c|c|c|c|c|c|c|c|}
\hline & \multicolumn{4}{|c|}{ Advice networks } & \multicolumn{4}{|c|}{ Personal discussion networks } \\
\hline & \multirow{2}{*}{$\frac{\text { Period } 1}{\text { Wave } 1}$} & \multirow{2}{*}{$\frac{\text { Period } 2}{\text { Wave } 2}$} & \multicolumn{2}{|c|}{ Period 3} & \multirow{2}{*}{$\frac{\text { Period } 1}{\text { Wave } 1}$} & \multirow{2}{*}{$\frac{\text { Period } 2}{\text { Wave } 2}$} & \multicolumn{2}{|c|}{ Period 3} \\
\hline & & & Wave 3 & Wave 4 & & & Wave 3 & Wave 4 \\
\hline Density & 0.051 & 0.053 & 0.061 & 0.056 & 0.025 & 0.034 & 0.035 & 0.041 \\
\hline \multicolumn{9}{|l|}{ Outdegree indices } \\
\hline Average degree & 3.71 & 3.84 & 4.41 & 4.05 & 1.79 & 2.48 & 2.51 & 2.98 \\
\hline Standard deviation & 5.45 & 3.40 & 3.91 & 3.53 & 2.05 & 2.27 & 2.44 & 2.79 \\
\hline Min & 0 & 0 & 0 & 0 & 0 & 0 & 0 & 0 \\
\hline Max & 38 & 15 & 16 & 19 & 9 & 13 & 13 & 13 \\
\hline \multicolumn{9}{|l|}{ Indegree indices } \\
\hline Average degree & 3.71 & 3.84 & 4.41 & 4.05 & 1.79 & 2.48 & 2.51 & 2.98 \\
\hline Standard deviation & 2.22 & 2.24 & 2.26 & 2.42 & 1.45 & 1.53 & 1.54 & 1.66 \\
\hline Min & 0 & 0 & 0 & 0 & 0 & 0 & 0 & 0 \\
\hline Max & 9 & 9 & 9 & 10 & 6 & 5 & 5 & 6 \\
\hline Number of ties & 241 & 242 & 269 & 247 & 116 & 156 & 153 & 182 \\
\hline Missing fraction & $11.0 \%$ & $13.7 \%$ & $16.4 \%$ & $16.4 \%$ & $11.0 \%$ & $13.7 \%$ & $16.4 \%$ & $16.4 \%$ \\
\hline Mutual dyads & 37 & 50 & 58 & 51 & 27 & 39 & 38 & 46 \\
\hline Asymmetric dyads & 167 & 142 & 153 & 145 & 62 & 78 & 77 & 90 \\
\hline \multicolumn{9}{|l|}{ Tie changes } \\
\hline Creating tie $(0 \rightarrow 1)$ & & 154 & 102 & 61 & & 80 & 44 & 44 \\
\hline Dissolving tie $(1 \rightarrow 0)$ & & 149 & 72 & 80 & & 38 & 36 & 27 \\
\hline Stable tie $(1 \rightarrow 1)$ & & 81 & 152 & 171 & & 67 & 96 & 120 \\
\hline Jaccard index & & 0.21 & 0.47 & 0.55 & & 0.36 & 0.55 & 0.63 \\
\hline
\end{tabular}

These statistics are reported in RSiena outputs, except for outdegree/indegree indices, mutual dyads, and asymmetric dyads calculated by the igraph package in $R$. Period refers to time between two waves (Periods 1 and 3 describe the transition during the semester and Period 2 describes the transition during the semester break). The total number of undergraduates who agreed to participate in the study and put their name in the roster is 73 . The missing fraction indicates the percentage of unit non-response at each wave.

absence) to 1 (tie presence) or vice versa (1-0) over time (Snijders et al., 2010). Modeling tie creation and maintenance (dissolution) has also become common in this framework (Sadewo et al., 2020).

SAOM allows us to directly examine the attribute and network effects on multiplex tie formation processes while controlling each effect. In addition, the examination of social network dynamics in cost-reward contexts is suitable for the assumptions of SAOM (Block et al., 2019). This study implemented the possible characteristics of individuals high in generalized trust described above within a framework of SAOM as a set of testable hypotheses. Details of the analysis are reported in Supplementary Material.

\section{RESULTS}

\section{Descriptive Statistics}

Two sets of four $73 \times 73$ adjacency matrices of directed graphs were created based on answers to questions for the advice and personal discussion networks across the four waves. A total of 42,048 cells in the full matrices $(=73$ actors $\times 73$ actors $\times 2$ networks $\times 4$ waves -584 diagonal elements) were objects of analysis. Table 1 presents the structural characteristics of the advice and personal discussion networks (for graphical depictions of each network, Supplementary Figures 1, 2; for the structural characteristics of the acquaintanceship and renown networks, Supplementary Table 2). Missing fractions were lower than $20 \%$ across the survey, which satisfied the minimum requirements of longitudinal network research to achieve reasonable parameter estimations (Huisman and Steglich, 2008). The average degree of ties did not drastically change across the four waves in these networks, indicating that actors maintained a similar number of ties in the networks.

In order to check the stability of successive networks, we calculated the Jaccard indices at each period by dividing the number of stable ties (i.e., ties that existed during the period) by the sum of the numbers of creating/dissolving ties (i.e., ties that changed during the period). For the application of SAOM to relatively small- or mediumsized network data, the Jaccard index should be larger than 0.20 (Ripley et al., 2019), and the current dataset satisfied that criterion. In both types of networks, the indices were low at Period 1 and rapidly increased at Period 2, and this trend corresponded with the increase in the number of mutual dyads. These tendencies indicate that the networks involved substantial changes in structure at the early stage of network evolution.

Figure 1 presents the average proportion of changes in network compositions from the first wave (Wave 1) to the subsequent waves (Waves 2-4) in the advice and the personal discussion networks. Individuals low in generalized trust showed relatively higher stability of advice ties across the waves than those middle or high in generalized trust. The patterns were similar in the advice and the personal discussion networks, probably due to the overlap of the two networks. This indicates the necessity of multiplex network analysis to examine the effect of generalized trust on tie formation in one network under controlling for the effect of the other network.

\section{Parameter Estimation}

\section{Generalized Trust and Cross-Network Effects}

Parameter estimates of the models for two uniplex networks (advice and personal discussion) and one multiplex network were 


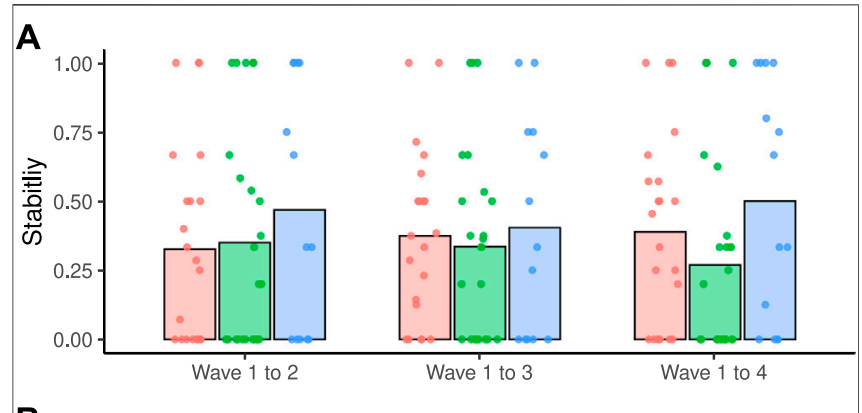

B

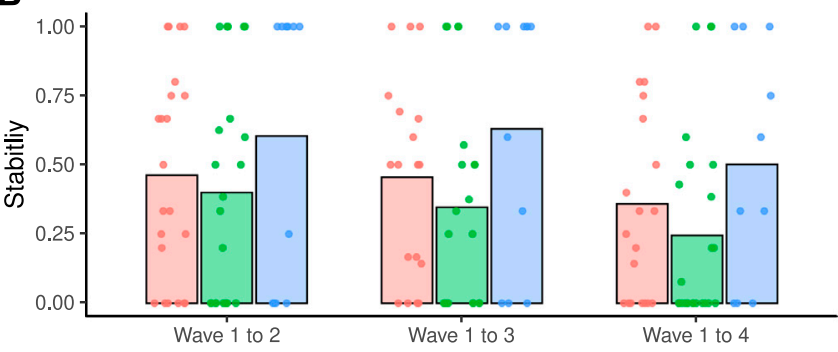

Generalized Trust $\square$ High $\square$ Medium $\square$ Low

FIGURE 1 | Generalized trust and changes in network compositions across the waves. (A) Advice network. (B) Personal discussion network. In both networks, stability indicates the proportion of ties at Wave 1 remained at the subsequent waves (Waves 2-4). Bars represent mean values and scatter plots represent the distributions of observed values. Individuals are classified into three groups according to their levels of generalized trust scores (ranging from 6 to $30 ; M=18.91, S D=4.74$ ). High: individuals high in generalized trust (scores 22 to 30 (larger than $M+0.5 S D$ ); $N=24$ ); Medium: individuals medium in generalized trust (scores 17 to 21 (within $M \pm 0.5 S D$ ); $N=28$ ); and L: individuals low in generalized trust (scores 6 to 16 (smaller than $M-0.5 S D) ; N=18)$.

separately conducted using RSiena (Ripley et al., 2019). All models satisfied convergence criteria (i.e., overall maximum convergence ratios $<0.25$ and mean absolute individual $t$-statistics for each parameter $<0.10$; Ripley et al., 2019), so the current estimated values were reliable and interpretable to understand network dynamics. The estimation results of trustrelated variables in the uniplex and multiplex analyses are shown in Table 2. The results of the models, including all variables, are presented in Supplementary Tables 3,4.

The effects of generalized trust in advice and personal discussion networks substantially varied in the forms of analyses. The uniplex analysis revealed that the following effects were significant only in the advice network: the negative endowment effect of generalized trust ego revealed that individuals high in generalized trust were more likely to terminate (i.e., not to maintain) existing advice ties than those low in generalized trust. The negative interaction effect between generalized trust ego and transitive closure (geometrically weighted edgewise shared partners; GWESP) had a diminishing weight on additional indirect paths for individuals high in generalized trust, indicating that individuals high in generalized trust were less likely to belong to closed triads than those low in generalized trust. The alter effect of generalized trust was positive and significant in the advice network. This indicates that actors high in generalized trust were more likely to be nominated by others who sought advice.

Meanwhile, generalized trust also showed significant influences on the creation of new ties not only in the adviceseeking network but also in the personal discussion network. The positive creation effect of generalized trust ego indicates that individuals high in generalized trust were more likely to create outgoing ties than those low in generalized trust. The resemblance of the trust-related tie-creation effects in both networks seems to be contradictory to the original predictions.

However, when the tie-formation processes across advice and personal discussion networks were taken into consideration in the multiplex analysis, the trust-related effects were significant only in the advice network, not in the personal discussion network. These findings give support to Hypotheses 1, 2, and 3 , in which the effects of generalized trust were expected to be found only in the advice network. No significant ego $\times$ alter effect of generalized trust means that homophily in generalized trust is not essential to explain tie changes in the current networks.

We create two figures to further understand the underlying patterns of advice tie formation in relation to generalized trust. Figure 2 shows the ego-alter selection plots for A) tie creation and B) endowment tendencies in the advice network. Individuals high in generalized trust were more likely to create new ties and less likely to maintain existing ties than those lower in generalized trust. The positive slope observed in both plots also indicates that individuals high in generalized trust were likely to be nominated by other individuals. Figure 3 shows the linear combinations of the trust-related transitive closure effect in the advice network. A) When the degree of local clustering (represented as the number of edgewise shared partners in the GWESP parameter) was small, generalized trust increased the likelihood of forming closed triads for advice seeking. In contrast, B) when the degree of local clustering was large, generalized trust decreased the weight to form closed triads.

In terms of cross-network effects, the interaction effects of generalized trust and the cross-network effects were incorporated in the model to test the possibility that individuals high in generalized trust are inclined to turn more to strangers than to friends for advice seeking, but none of the effects were significant. Therefore, Hypothesis 4 was not supported. In the meantime, positive trends were observed in the outgoing personal-discussion-to-advice effect for tie creation and endowment. Personal discussion ties were likely to induce and stabilize advice-seeking ties in the same dyad.

Extraversion-related effects were not significant (Supplementary Table 4), confirming that the aforementioned networking processes stemmed from the characteristics of generalized trust.

\section{Wald-type Tests}

To check if the trust-related effects are substantial in the model in the multiplex analysis, we performed multi-parameter Wald-type tests for the objective function effects of generalized trust (creation ego, endowment ego, alter, ego $\times$ alter, generalized 
TABLE 2 | Parameter estimates of generalized trust in stochastic actor-oriented models.

\begin{tabular}{|c|c|c|c|c|}
\hline & \multicolumn{2}{|c|}{ Uniplex network } & \multicolumn{2}{|c|}{ Multiplex networks } \\
\hline & Advice & Personal discussion & Advice & Personal discussion \\
\hline \multicolumn{5}{|l|}{ Actor-level effects } \\
\hline Trust alter & $0.035(0.011)^{\star \star}$ & $0.033(0.018)$ & $0.032(0.012)^{\star \star}$ & $0.007(0.031)$ \\
\hline Trust ego (endowment) & $-0.498(0.097)^{\star \star \star}$ & $-0.276(0.174)$ & $-0.509(0.104)^{\star \star \star}$ & $-0.770(0.912)$ \\
\hline Trust ego (creation) & $0.669(0.107)^{\star \star \star}$ & $0.415(0.153)^{\star \star}$ & $0.640(0.112)^{\star \star \star}$ & $1.200(1.161)$ \\
\hline Trust ego $\times$ trust alter & $-0.001(0.002)$ & $-0.001(0.004)$ & $-0.001(0.003)$ & $0.005(0.006)$ \\
\hline Trust ego $\times$ GWESP closure & $-0.075(0.033)^{\star}$ & $-0.092(0.052)$ & $-0.069(0.035)^{\star}$ & $-0.100(0.127)$ \\
\hline \multicolumn{5}{|l|}{ Network effects } \\
\hline Reciprocity & $2.755(0.262)^{\star \star \star}$ & $3.887(0.465)^{\star \star \star}$ & $2.417(0.304)^{\star \star \star}$ & $3.994(0.854)^{\star \star \star}$ \\
\hline GWESP closure & $2.201(0.174)^{\star \star \star}$ & $2.514(0.255)^{\star \star \star}$ & $2.055(0.184)^{\star \star \star}$ & $2.205(0.488)^{\star \star \star}$ \\
\hline GWESP $\times$ reciprocity & $-0.520(0.312)$ & $-0.572(0.505)$ & $-0.914(0.342)^{\star \star}$ & $-0.332(1.002)$ \\
\hline \multicolumn{5}{|l|}{ Cross-network effects } \\
\hline Outgoing personal discussion to advice (endowment) & & & $1.711(0.571)^{\star \star}$ & \\
\hline Outgoing personal discussion to advice (creation) & & & $1.089(0.390)^{\star *}$ & \\
\hline Outgoing advice to personal discussion (endowment) & & & $-0.801(1.013)$ & \\
\hline Outgoing advice to personal discussion (creation) & & & $6.931(3.584)$ & \\
\hline Trust ego $\times$ outgoing personal discussion to advice (endowment) & & & $-0.007(0.169)$ & \\
\hline Trust ego $\times$ outgoing personal discussion to advice (creation) & & & $0.239(0.155)$ & \\
\hline Trust ego $\times$ outgoing advice to personal discussion (endowment) & & & $0.277(0.313)$ & \\
\hline Trust ego $\times$ outgoing advice to personal discussion (creation) & & & $-0.681(0.651)$ & \\
\hline
\end{tabular}

${ }^{* * *} p<.001,{ }^{* *} p<.01,{ }^{*} p<.05$; Standard errors are presented in parentheses; GWESP = geometrically weighted edgewise shared partners; Overall maximum convergence ratios = 0.09 (uniplex advice network), 0.13 (uniplex personal discussion network), and 0.24 (multiplex advice and personal discussion networks). Complete results including other variables are available in Supplementary Material.

trust $\times$ transitive closure, and cross-network effects of generalized trust) in the advice and personal discussion networks, respectively. The result was significant in the advice network $\left(\chi^{2}[8]=63.1, p<0.001\right)$, but not in the personal discussion network $\left(\chi^{2}[8]=5.59, p=0.69\right)$, suggesting that the dynamics of the former network substantially depend on generalized trust.

\section{Goodness of Fit}

The goodness-of-fit statistics for the multiplex analysis were reported in Supplementary Figures 3, 4 separately for the advice and personal discussion networks. Overall, the model fit well to the data, $p s>0.10$, indicating non-significance of the Mahalanobis distance between the observed and the simulated data, except for the outdegree distribution in the personal discussion network $(p<0.05)$. Although the finding is not precise enough to ensure the equality of the outdegree distributions between the simulated and observed data, the violin plots do not show a considerable discrepancy between the two. We therefore conclude that the current model is generally acceptable in that the network statistics simulated from the estimated model do not substantially deviate from those found in the observed data.

\section{DISCUSSION}

Based on the emancipation theory of trust, this study investigated the role of generalized trust in the social selection processes among first-year undergraduates. The multiplex analysis in SAOM revealed that individuals high in generalized trust tended to terminate existing ties, create new ties, and form open triads more in the advice network than in the personal discussion network. The findings provide empirical support for the initial predictions, positing that generalized trust is negatively related to the tendency for the maintenance of existing advice ties (Hypothesis 1), positively related to the tendency for the creation of outgoing advice ties (Hypothesis 2), and negatively related to the tendency for the formation of closed triads (Hypothesis 3), respectively. We also made a prediction at the cross-network level that individuals high in generalized trust tend to diminish the weight of personal discussion ties when creating advice-seeking ties in the same dyads (Hypothesis 4), but this was not supported. Homophily in generalized trust was not verified.

The multiplex analysis also yielded several unanticipated trustrelated effects in the advice network. Individuals high in generalized trust were likely to receive more nominations from others than those low in generalized trust. They were also likely to form closed triads when the degree of local clustering was small (Figure 3A). Considering together with the findings mentioned above, individuals high in generalized trust may be not lone wolves who care little about groups and pursue only their own interests but great strategists who excel in networking. They use different networking strategies depending on the degree of local clustering. They do take account of being embedded in small clusters for seeking advice, but at the same time, they prefer not to take part in large clusters and try to expand their advice ties outside the boundary so that they can get access to valuable information. Their balanced positioning in the advice network would make them more resourceful and attract others.

Initially, trust-related effects were observed in both the advice and personal discussion networks in the uniplex analysis. To understand the logic behind the networking strategy of individuals high in generalized trust, we should first mention the indistinctiveness of the role of generalized trust in the advice and personal discussion networks in that analysis. This would be elucidated in terms of the ambiguity of relationship commitment 

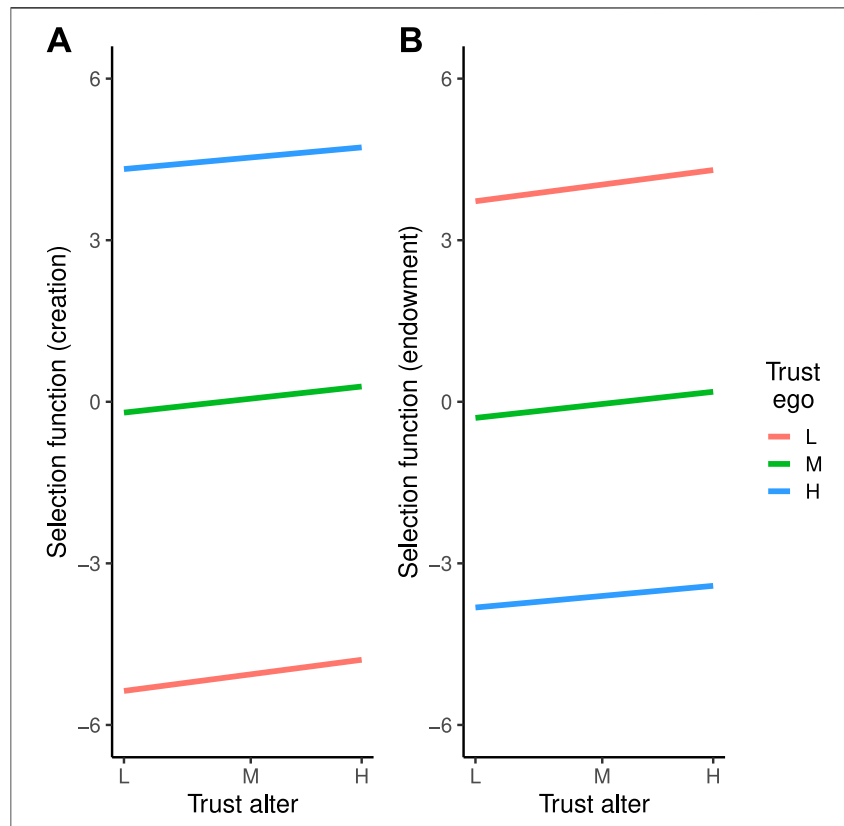

FIGURE 2 | Ego-alter selection plot for the effect of generalized trust on (A) the creation and (B) the endowment of advice ties for the model in Table 2 [including Trust ego (creation or endowment), Trust alter, and Trust ego $\times$ alter]. The lines indicate social selection functions for three levels of ego's generalized trust [high (score = 26), medium (score = 19), and low (score =11)] as a function of alter's generalized trust (on the $x$-axis).

in the current research. This study used communication content (advice seeking and self-disclosure) to distinguish between lowand high-commitment relationships. However, the level of relationship commitment is determined not only by the context of communication but also by the duration of relationships. For example, a nation-level survey on the impact of generalized trust on social support exchange (Ishiguro and Okamoto, 2013) operationally defined the length of time a relationship was maintained as an index of relationship commitment and found that the longer the duration of relationships among non-kin, the larger the amount of social support provision for the relationships. In this study, most of the personal discussion ties would be developed and maintained only for a short term after matriculation. This propensity may weaken commitment to the ties for personal discussion and promote individuals high in generalized trust to create new ties in order to find better conversation partners in the network. If this were the only case, we could have argued the validity of the emancipation theory of trust in low commitment relationships.

However, when considering the findings of the multiplex analysis, the mechanism is more complicated than originally expected. Remember that the parameters of SAOM describe patterns of possible tie changes explicitly provided to each actor in a network. We found a tendency to have personal discussion ties to induce advice seeking in the same dyads in the multiplex analysis when the parameters of cross-network tie formation were included in the model. This means that resources for personal discussion become embedded in advice-seeking
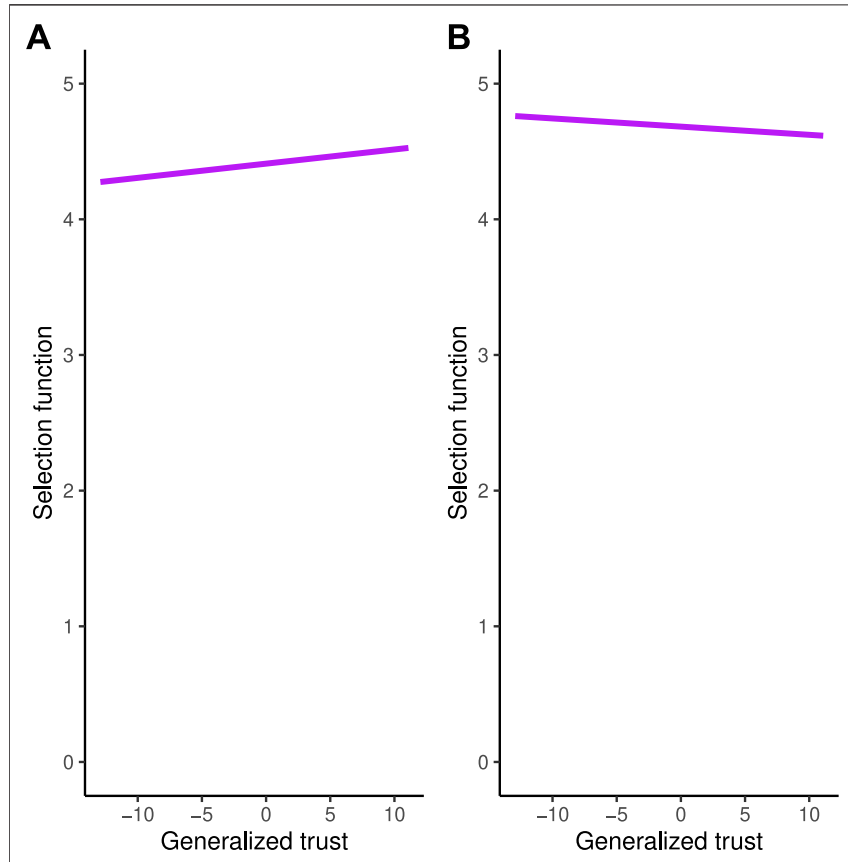

FIGURE 3 | Linear combination plots of the effect of generalized trust on the formation of transitive closure for advice seeking for the model in Table 2 (including reciprocity, GWESP closure, GWESP closure $\times$ reciprocity, Trust ego × GWESP closure). (A) The degree of local clustering (the number of outgoing two-path) $=3$. (B) The degree of local clustering $=8$. GWESP $=$ geometrically weighted edgewise shared partners. $\alpha=0.69$ in GWESP closure effects.

social circles through the dynamics of network evolution. The process is interpreted as backward relationship reinforcements after close ties are established.

In order to understand the findings of the multiplex analysis, it is helpful to reconceptualize tie multiplexity as a valid indicator of high-commitment relationships (Verbrugge, 1979; Burt, 1980; Kadushin, 2012) based on the strong convergent trend toward the tie overlap between the advice and personal discussion networks. It is natural for first-year undergraduates to be strongly incentivized to find safe haven in their new community through the construction of social ties connected in multiple ways. The prevalence of tie multiplexity would make actors in a network feel hesitant to cut and renew their existing advice ties due to psychological closeness built through the same ties. The lack of evidence in cross-network tie-formation effects triggered by generalized trust indicates that even individuals high in generalized trust value tie multiplexity once such relationships are established.

In this research, we measured generalized trust by Yamagishi's psychological scale. Some may think that an experimental approach, such as the trust game, is a better alternative with the attitudinal measure. However, a recent extensive review on the measurement of trust (Alos-Ferrer and Farolfi, 2019) points out the limitations of the trust game paradigm due to its confounding with prosocial and risk orientations and low stability. In other words, the abstract concept of generalized trust can be captured by various behavioral/survey indicators, 
and no specific methodology covers all aspects of the intuitive notions of trust. There is also research reporting a risk-trust confound in the trust game (Chetty et al., 2020), so controlling risk aversion would be important to adjust the power of trustrelated variables on the prediction of trust-related behavior.

The findings also provide significant implications for organizational management. In organizations with dynamic capabilities (Teece et al., 1997), establishing novel knowledge assets is essential to facilitate innovation for survival in an uncertain social world. Individuals high in generalized trust would play a key role in the process: they would get better access to valuable resources embedded in organizational contexts and become popular and advantageous among coworkers owing to their rational tie-formation and dissolution strategies to integrate and reconstruct the value of the resources. It would also be helpful if stakeholders understand the importance of social network dynamics driven by generalized trust to break the bonds of convention in established organizations.

The current research is the first to demonstrate the applicability of the emancipation theory of trust to the process of multiple network dynamics. Meanwhile, we could also point out several limitations in the current study. This study analyzed complete network data where the network boundary was set to a department of a university. However, such a specific focus on a network boundary excludes different groups and contexts and narrows the coverage of each actor's personal discussion activity. This may cause a potential lack in capturing the entire process of social selection. The personal/egocentric network approach allows respondents to nominate anyone who has direct connections with them both inside and outside a specific boundary of a reference group to which they mainly belong. Applying this method through snowball sampling makes it easy for researchers to manipulate social selection processes in consideration of the diversity of network composition across different social contexts. Future research should test the validity of the emancipation theory of trust from the personal/ egocentric network perspective.

It is also important to emphasize that the emancipation theory of trust focuses on a de-personalized concept of generalized trust rather than a sociometric assessment of interpersonal trust (e.g., "Who do you trust?") that has been more common in the social network domain (Burt and Knez, 1996; Levin and Cross, 2004; Shakya et al., 2020). The former represents an actor's default expectation of trustworthiness of people in general based on positive belief and knowledge about humans, whereas the latter represents an actor's expectation of trustworthiness of particular others based on their positive attitudes and feelings toward the actor. Although interpersonal trust is beyond the scope of the current study, Yamagishi (2011) suggests that the process of social network formation based on interpersonal trust is the exact opposite of that based on generalized trust. Actors having many ties with interpersonal trust would have secure feelings with relational embeddedness, but these existing ties would also work like a ball and chain, preventing them from moving on to new ties that could offer better benefits. Therefore, it is predicted that actors having many ties based on interpersonal trust do not show a strong tendency to switch ties. More concretely, actors nominating a greater number of other particular actors as trustworthy might be less likely to create new ties and terminate existing ties and to hold open triads in their advice and personal discussion networks due to their preference for relational embeddedness in their existing social circle (also see Yosano and Hayashi, 2005 for more discussion). Future research should consider the dynamic interplay between dispositional (generalized) and sociocentric (interpersonal) trust in a framework of network evolution.

There is the fact that individuals high in generalized trust are not a majority in the overall population. A descriptive summary of the World Values Survey database 2010-2014 (World Values Survey Association, 2015) reports that the mean percentage of survey respondents who chose "Most people can be trusted" over "Need to be very careful" is only $24.5 \%(S D=15.7)$ across 59 nations $(N=86,274)$, and $35.9 \%$ in Japan $(N=2,443)$. Given this, the opportunity for those low in generalized trust to increase their trustfulness through encounters with others high in generalized trust is limited. Future research should examine effective ways to establish benevolence across a whole social network.

\section{DATA AVAILABILITY STATEMENT}

The raw data supporting the conclusion of this article will be made available by the authors, without undue reservation.

\section{ETHICS STATEMENT}

The studies involving human participants were reviewed and approved by the Ethical Review Board of the Graduate School of Education and Human Development, Nagoya University. The patients/participants provided their written informed consent to participate in this study.

\section{AUTHOR CONTRIBUTIONS}

TI and TH designed and performed research; TI analyzed data; and $\mathrm{TI}$ and $\mathrm{TH}$ wrote the paper.

\section{FUNDING}

The study was supported by JSPS KAKENHI Grant Number JP25780371 to TI. The funder had no role in study design, data collection and analysis, decision to publish, or preparation of the manuscript. 


\section{ACKNOWLEDGMENTS}

We thank Johan Koskinen and Yang Li for their helpful comments on an earlier version of the manuscript and Jin Kato and Ryuichi Tamai for their assistance in collecting data.

\section{REFERENCES}

Alos-Ferrer, C., and Farolfi, F. (2019). Trust Games and beyond. Front. Neurosci. 13, 887. doi:10.3389/fnins.2019.00887

Baumeister, R. F., and Leary, M. R. (1995). The Need to Belong: Desire for Interpersonal Attachments as a Fundamental Human Motivation. Psychol. Bull. 117, 497-529. doi:10.1037/0033-2909.117.3.497

Block, P., Stadtfeld, C., and Snijders, T. A. B. (2019). Forms of Dependence: Comparing SAOMs and ERGMs from Basic Principles. Sociological Methods Res. 48 (1), 202-239. doi:10.1177/0049124116672680

Bonnefon, J.-F., Hopfensitz, A., and De Neys, W. (2013). The Modular Nature of Trustworthiness Detection. J. Exp. Psychol. Gen. 142, 143-150. doi:10.1037/ a0028930

Bravo, G., Squazzoni, F., and Boero, R. (2012). Trust and Partner Selection in Social Networks: An Experimentally Grounded Model. Social Networks 34, 481-492. doi:10.1016/j.socnet.2012.03.001

Burt, R. S., and Knez, M. (1996). "Trust and Third-Party Gossip," in Trust in Organizations: Frontiers of Theory and Research. Editors R. M. Kramer and T. R. Tyler (Thousand Oaks: CA), 68-89.

Burt, R. S. (1980). Models of Network Structure. Annu. Rev. Sociol. 6, 79-141. doi:10.1146/annurev.so.06.080180.000455

Burt, R. S. (1993). Structural Holes: The Social Structure of Competition. Cambridge, MA: Harvard University Press.

Chetty, R., Hofmeyr, A., Kincaid, H., and Monroe, B. (2020). The Trust Game Does Not (Only) Measure Trust: The Risk-Trust Confound Revisited. J. Behav. Exp. Econ. 90, 101520 doi:10.1016/j.socec.2020.101520

Coleman, J. S. (1988). Social Capital in the Creation of Human Capital. Am. J. Sociol. 94, 95-120. doi:10.1086/228943

Davis, J. A. (1970). Clustering and Hierarchy in Interpersonal Relations: Testing Two Graph Theoretical Models on 742 Sociomatrices. Am. Sociological Rev. 35, 843-851. doi:10.2307/2093295

Dunbar, R. I. M., Korstjens, A. H., and Lehmann, J. (2009). Time as an Ecological Constraint. Biol. Rev. Cambridge Phil. Soc. 84, 413-429. doi:10.1111/j.1469185x.2009.00080.x

Erickson, B. H. (1988). "The Relational Basis of Attitudes," in Social Structures: A Network Approach. Editors B. Wellman and S. D. Berkowitz (New York: Cambridge University Press), 99-121.

Foa, E. B., and Foa, U. G. (1976). "Resource Theory of Social Exchange," in Contemporary Topics in Social Psychology. Editors J. W. Thibaut, J. T. Spence, and R. C. Carson (Morristown, NJ: General Learning Press).

Granovetter, M. S. (1973). The Strength of Weak Ties. Am. J. Sociol. 78, 1360-1380. doi:10.1086/225469

Hashimoto, H., Maeda, K., Tomida, S., and Tanida, S. (2020). The Association between the Level of General Trust and the Judgment Accuracy of Group Members' Cooperation in a Social Dilemma. Lett. Evol. Behav. Sci. 11, 27-30. doi:10.5178/lebs.2020.77

Hayashi, N., and Yamagishi, T. (1998). Selective Play: Choosing Partners in an Uncertain World. Pers Soc. Psychol. Rev. 2, 276-289. doi:10.1207/ s15327957pspr0204_4

Huisman, M., and Steglich, C. (2008). Treatment of Non-response in Longitudinal Network Studies. Soc. Networks 30, 297-308. doi:10.1016/j. socnet.2008.04.004

Igarashi, T., Kashima, Y., Kashima, E. S., Farsides, T., Kim, U., Strack, F., et al. (2008). Culture, Trust, and Social Networks. Asian J. Soc. Psychol. 11, 88-101. doi:10.1111/j.1467-839x.2007.00246.x

Ishiguro, I., and Okamoto, Y. (2013). Two Ways to Overcome Social Uncertainty in Social Support Networks: A Test of the Emancipation Theory of Trust by Comparing Kin/nonkin Relationships. Jpn. Psychol. Res. 55, 1-11. doi:10.1111/j. 1468-5884.2012.00536.x

\section{SUPPLEMENTARY MATERIAL}

The Supplementary Material for this article can be found online at: https://www.frontiersin.org/articles/10.3389/fcomm.2021.667082/ full\#supplementary-material

Kadushin, C. (2012). Understanding Social Networks: Theories, Concepts, and Findings. New York: Oxford University Press.

Levin, D. Z., and Cross, R. (2004). The Strength of Weak Ties You Can Trust: The Mediating Role of Trust in Effective Knowledge Transfer. Manag. Sci. 50, 1477-1490. doi: $10.1287 / \mathrm{mnsc} .1030 .0136$

Lusher, D., Robins, G., Pattison, P. E., and Lomi, A. (2012). “Trust Me”: Differences in Expressed and Perceived Trust Relations in an Organization. Soc. Networks 34, 410-424. doi:10.1016/j.socnet.2012.01.004

Mcevily, B., and Zaheer, A. (1999). Bridging Ties: A Source of Firm Heterogeneity in Competitive Capabilities. Strat. Mgmt. J. 20, 1133-1156. doi:10.1002/(sici) 1097-0266(199912)20:12<1133::aid-smj74>3.0.co;2-7

Mcpherson, M., Smith-Lovin, L., and Cook, J. M. (2001). Birds of a Feather: Homophily in Social Networks. Annu. Rev. Sociol. 27, 415-444. doi:10.1146/ annurev.soc.27.1.415

Putnam, R. D. (2000). Bowling Alone: The Collapse and Revival of American Community. New York: Simon \& Schuster. doi:10.1145/358916.361990

Rand, D. G., Arbesman, S., and Christakis, N. A. (2011). Dynamic Social Networks Promote Cooperation in Experiments with Humans. Proc. Natl. Acad. Sci. 108, 19193-19198. doi:10.1073/pnas.1108243108

Ripley, R. M., Snijders, T. A. B., Boda, Z., Vörös, A., and Preciado, P. (2019). Manual for RSiena. Nuffield College: University of Oxford, Department of Statistics.

Sadewo, G. R. P., Kashima, E. S., Gallagher, C., Kashima, Y., and Koskinen, J. (2020). International Students' Cross-Cultural Adjustment: Social Selection or Social Influence?. J. Cross-Cultural Psychol. 51, 490-510. doi:10.1177/ 0022022120930092

Sampson, R. J., Raudenbush, S. W., and Earls, F. (1997). Neighborhoods and Violent Crime: A Multilevel Study of Collective Efficacy. Science 277, 918-924. doi:10.1126/science.277.5328.918

Shakya, H. B., Challa, S., Nouhou, A. M., Vera-Monroy, R., Carter, N., and Silverman, J. (2020). Social Network and Social Normative Characteristics of Married Female Adolescents in Dosso, Niger: Associations with Modern Contraceptive Use. Glob. Public Health. doi:10.1080/17441692.2020.1836245

Snijders, C., and Keren, G. (2001). "Do you Trust? Whom Do You Trust? when Do You Trust?," in Advances in Group Processes (Emerald Group Publishing Limited), 129-160.

Snijders, T. A. B. (2017). Siena Advanced Users' Meeting 2017. The 9th Winter School on Longitudinal Social Network Analysis and the 2017 Advanced Siena Users Meeting. Norrköping, Sweden: institute for analytical sociology.

Snijders, T. A. B. (1996). Stochastic Actor-oriented Models for Network Change. J. Math. Sociol. 21, 149-172. doi:10.1080/0022250x.1996.9990178

Snijders, T. A. B., van de Bunt, G. G., and Steglich, C. E. G. (2010). Introduction to Stochastic Actor-Based Models for Network Dynamics. Soc. Networks 32, 44-60. doi:10.1016/j.socnet.2009.02.004

Sturgis, P., Patulny, R., Allum, N., and Buscha, F. (2015). "Social Connectedness and Generalized Trust: a Longitudinal Perspective," in Handbook of Research Methods and Applications in Social Capital. Editor Y. Li (Cheltenham, UK: Edward Elgar Publishing)), 76-90.

Teece, D. J., Pisano, G., and Shuen, A. (1997). Dynamic Capabilities and Strategic Management. Strat. Mgmt. J. 18, 509-533. doi:10.1002/(sici)10970266(199708)18:7<509::aid-smj882>3.0.co;2-z

Valenzuela, S., Park, N., and Kee, K. F. (2009). Is There Social Capital in a Social Network Site?: Facebook Use and College Students' Life Satisfaction, Trust, and Participation. J. Computer-Mediated Commun. 14, 875-901. doi:10.1111/j. 1083-6101.2009.01474.x

van de Bunt, G. G., van Duijn, M. A. J., and Snijders, T. A. B. (1999). Friendship Networks through Time: An Actor-Oriented Dynamic Statistical Network Model. Comput. Math. Organ. Theor. 5, 167-192. doi:10.1023/a: 1009683123448

Verbrugge, L. M. (1979). Multiplexity in Adult Friendships. Social Forces 57, 1286-1309. doi:10.2307/2577271 
Watabe, M., Ban, H., and Yamamoto, H. (2011). Judgments about Others' Trustworthiness: An fMRI Study. Lett. Evol. Behav. Sci. 2, 28-32. doi:10. 5178/lebs.2011.16

Weidman, J. (1989). "Undergraduate Socialization: A Conceptual Approach," in Higher Education: Handbook of Theory and Research. Editor J. C. Smart (New York: Agathon Press), 289-322.

World Values Survey Association (2015). World Values Survey Wave 6 2010-2014 Official Aggregate v.20150418 [Online]. Madrid, Spain: Aggregate File Producer: Asep/JDS. Available: http://www.worldvaluessurvey.org/ [Accessed February 8, 2021].

Yamagishi, T. (2011). Trust: The Evolutionary Game of Mind and Society. Tokyo: Springer. doi:10.1007/978-4-431-53936-0

Yamagishi, T., Akutsu, S., Cho, K., Inoue, Y., Li, Y., and Matsumoto, Y. (2015). Two-component Model of General Trust: Predicting Behavioral Trust from Attitudinal Trust. Soc. Cogn. 33, 436-458. doi:10.1521/soco.2015.33.5.436

Yamagishi, T., Cook, K. S., and Watabe, M. (1998). Uncertainty, Trust, and Commitment Formation in the United States and Japan. Am. J. Sociol. 104, 165-194. doi:10.1086/210005

Yamagishi, T., Kikuchi, M., and Kosugi, M. (1999). Trust, Gullibility, and Social Intelligence. Asian J. Soc. Psychol. 2, 145-161. doi:10.1111/1467-839x.00030
Yamagishi, T., and Yamagishi, M. (1994). Trust and Commitment in the United States and Japan. Motiv. Emot. 18, 129-166. doi:10.1007/bf02249397

Yosano, A., and Hayashi, N. (2005). Social Stratification, Intermediary Groups and Creation of Trustfulness. Sociological Theor. Methods 20, 27-44. doi:10.11218/ ojjams.20.27

Yoshimoto, T., and Hasegawa, A. (2017). How Does General Trust Improve Mental Health of University Students? A Study Based on the Emancipation Theory of Trust. Jjre 24, 92-100. doi:10.4092/jsre.24.2_92

Conflict of Interest: The authors declare that the research was conducted in the absence of any commercial or financial relationships that could be construed as a potential conflict of interest.

Copyright $\odot 2021$ Igarashi and Hirashima. This is an open-access article distributed under the terms of the Creative Commons Attribution License (CC BY). The use, distribution or reproduction in other forums is permitted, provided the original author(s) and the copyright owner(s) are credited and that the original publication in this journal is cited, in accordance with accepted academic practice. No use, distribution or reproduction is permitted which does not comply with these terms. 